\title{
Acknowledgement to Reviewers of Brain Sciences in 2017
}

Brain Sciences Editorial Office

MDPI AG, St. Alban-Anlage 66, 4052 Basel, Switzerland

Published: 10 January 2018

Peer review is an essential part in the publication process, ensuring that Brain Sciences maintains high quality standards for its published papers. In 2017, a total of 160 papers were published in the journal. Thanks to the cooperation of our reviewers, the median time to first decision was 26 days and the median time to publication was 58.5 days. The editors would like to express their sincere gratitude to the following reviewers for their time and dedication in 2017:

\begin{tabular}{ll} 
Abhishek Desai & Anna Fogdell-Hahn \\
Adel Helmy & Anna J. Esbensen \\
Adrian Parke & Anne Beemelmanns \\
Agnieszka Fiszer & Anne Dezetter \\
Akassoglou Katerina & Anouk Y. J. M. Smeets \\
Akiko Tamasaki & Anthony Akkari \\
Alan Dorval & Anthony R. Mawson \\
Alan G. Finkel & Antonella Scorziello \\
Alan Winston & Antonino F. Germano \\
Alcy R. Torres & Antonio De Tanti \\
Alejandro López Tobón & Apit Hemakom \\
Alessandro Didonna & Armin Fuchs \\
Alessandro Tonacci & Arthur A. Vandenbark \\
Alexander U. Brandt & Ashraf S. Gorgey \\
Allen L. Ho & Athanasia Mouzaki \\
Amanda M. Brown & Atsuhiro Nakagawa \\
Amanda Seidl & Balapal S. Basavarajappa \\
Amy Mandaville & Barbara S. Koppel \\
Ana Ledo & Barbara Tettenborn \\
André A. Fenton & Bengt Källén \\
Andre Strydom & Bilal Khokhar \\
Andreas Luft & Boris Kotchoubey \\
Andreas Schulze & Brendan Rooney \\
Andrej Kral & Brent Kious \\
Andres M. Lozano & Brian Christie \\
Andrew Bagshaw & Brian Nils Lundstrom \\
Andrew C. Talk & Brooke N. Macnamara \\
Andrew J. Solomon & Bruce Lyeth \\
Andrew Post & Bruno Meloni \\
Andriy Temko & Bryan Duckham \\
Ángel Romero Martínez & Bryan Howell \\
Angela Narayan & Calvin Li \\
Anita Riecher-Rössler & Cameron B. Jeter \\
Ann B. Ragin & Camila Aquino \\
& \\
\hline &
\end{tabular}


Carlotta Zanaboni Dina

Carmela Bravaccio

Carmelo Lucio Sturiale

Caroline Junge

Catherine S. Tamis-LeMonda

Cecilia Giulivi

Cédric Galera

Charles A. Nelson

Chi-Chang Huang

Chloe Lane

Christian P. Müller

Christian Salvatore

Christine Winter

Christopher Miller

Christopher-James Harvey

Claire O'Callaghan

Clare Harrop

Claudia Maennel

Claudio Liguori

Clea Warburton

Clement Hamani

Clodagh Nolan

Cynthia R. Gross

Damian A. Stanley

Damian Jenkins

Damir Janigro

Daniel Cohen

Daniel Peterson

Daniel Swingley

Dario D. Lofrumento

Dario Siniscalco

David Bar-Or

David Facal

David M. Fergusson

David M. Schnyer

David P. Gavin

David R. Shprecher

David T. Denhardt

David W. Evans

David Wagner

David Ziegler

Davide Barbagallo

Deane Aikins

DeAnna L. Adkins

Deborah Ferguson

Denise Gobert

Denise Sharon

Dennis W. Vane

Devanshi Seth

Devin W. McBride

Dhanabalan Murali

Diego Torres-Russotto
Domenico De Berardis

Domenico Servello

Dominic Cheng

Dora Lozsadi

Douglas Weldon

Dwight Pierce

E. Richard Stanley

Eberhard Uhl

Edward V. Quadros

Edward Vul

Egilius L. H. Spierings

Eiichi Suehiro

Eling De Bruin

Elizabeth A. Strickland

Emily D. Ricketts

Emily Edmonds

Emily S. Nichols

Eric Braverman

Eric Glasgow

Eric Verin

Erik Sistermans

Erika Skoe

Erwin B. Montgomery

Eugene Park

Fabiana Geraci

Fabio Campanella

Fabio Di Domenico

Farid Rahimi

Felix Jimenez-Rondan

Felix Septianto

Feng Geng

Feng Gu

Feng Vankee Lin

Fernando L. Vale

Filmer Chu

Firas Bannout

Florence Gressier

Florence Levy

Florien Boele

Florin Despa

Francesco Bartoli

Francesco Carlo Morabito

Francesco Rigoli

Francisca S Rodriguez

Francisca S. Rodriguez

Franco Gemignani

Frank Flemisch

Frédéric Causeret

Frederik Steyn

Fredrik Karlsson

Friedemann Paul

Fulvio Lauretani 
Gabriel G. De La Torre

Gabriela G. Werner

Ganesh Naik

Garnett P. McMillan

Gary Simon

Gayannée Kedia

Geon Ho Bahn

Georg Widhalm

George Chrousos

George Opie

Georgios Paslakis

Gholson Lyon

Giacomo Vivanti

Gianluca Serafini

Giordano D'Urso

Giovanni Assenza

Giri Kumar Chandaka

Glen Bergeron

Gordon Harper

Gregory Fricchione

Gregory Heath

Gregory P. Mueller

Guennadi Saiko

Gurudutt Pendyala

H. Lee Swanson

Hans Van Der Steen

Hans-Gert Bernstein

Harrison C. Walker

Hasse Walum

Heather Bowling

Hideya Kodama

Hiran Thabrew

Hiroaki Wakimoto

Hirokazu Doi

Holly Bowen

Hon Wah Lee

Hong Jiao

Hovagim Bakardjian

Howard Chertkow

Howard Prentice

Ilya Bezprozvanny

Inga D. Neumann

Ingvild Saksvik-Lehouillier

Isaac T. Schiefer

Itoh Yasuhiro

J. Leigh Leasure

Jacqueline E. Reznik

Jaishankar Bharatharaj

Jake Neumann

James Adams

James O'Callaghan

James R. Couch
Jannette Rodríguez-Pallares

Jared W. Young

Javier Ortuño-Sierra

Jayashri Ghosh

Jean R. Hughes

Jean-Philippe Langevin

Jennifer Beer

Jennifer L. Lyons

Jennifer Rodger

Jeroen L. A. Pennings

Jérôme Brunelin

Jérôme Devy

Jerzy Bodurka

Jessica Robin

Jia Cheng

Jie Shi

Jill Crittenden

Jinyuan Wang

Joachim Fandrey

Jodi M Heaps

Joerg Wischhusen

Johanna Bick

John Gruzelier

John L. Woodard

John Magnotti

John Rolston

Jon Henner

Joohyung Lee

José Rouillard

Josep Esteve-Romero

Josephine Barnes

Joshua Park

Judith S. Miller

Julie A. Grieco

Julie Gros-Louis

Julio Ortega

Jyoti Mishra

Karolina Moustopoulou

Kate Karelina

Kathryn M. Yorkston

Kathy Carter

Kathy Mountjoy

Katsuhiko Nishimori

Katsura Takano

Kavitha Yaddanapudi

Kaylah Lalonde

Kevin C. Brennan

Kevin J. Black

Kevin Schalinskie

Kirsten R. Müller-Vahl

Kylie Bailey

Laraine Winter 
Larisa Poluektova

Larry Abel

Lee Campbell

Leena Mohapatra

Leher Singh

Leonard Verhagen Metman

Lesley Jones

Leslie Swanson

Leyan $\mathrm{Xu}$

Lili-Naz Hazrati

Lin Zhu

Lindsay D. Nelson

Lisa Wise-Faberowski

Lise Eliot

Litofsky N. Scott

Lora Talley Watts

Loren Marulis

Lori-Ann R. Sacrey

Luca Casartelli

Luis R. Peraza

Lyn O'Grady

Lynn Ulatowski

Madelyn Ray

Marcel Giezen

Marcello Ciaccio

Marco Carotenuto

Marcus Chen

Marcus Grimm

Maria Cabello

Maria Chahrour

Maria Chiara Buscarinu

Maria Christina Sergaki

Maria Hernandez

Maria Luca

Mariko Saito

Marion Ehrich

Marita Pruessner

Mark B. Detweiler

Mark R. N. Kotter

Markus Wettstein

Martha Michalkiewicz

Martijn Baart

Martin Gerbert Frasch

Martin Spüler

Martina Ardizzi

MARVIN R. Diaz

Mary Motz

MATTHEW ROBSON

Matthias Hohmann

Meera E. Modi

Melanie Morrison

Melanie Pina
Melissa Barber

Melissa Raven

Michael A. Ben-Avie

Michael D. Rawlins

Michael Johnston

Michael Perlis

Michael Schaefer

Michael Yoong

Michal Mielcarek

Michel Billiard

Mila Vulchanova

Mohd. Farooq Shaikh

Mohtashem Samsam

Montserrat Zurrón

Monty Silverdale

Myra Fernandes

Nafisa M. Jadavji

Nathanael J. Yates

Navkiran Kalsi

Nawei Sun

Nesha Burghardt

Niccolò Mora

Nicholas Altieri

Nicole Osier

Nikolaos Ziogas

Nnamdi Pole

Noémie Auclair Ouellet

Noman Naseer

Nora Vanegas Arroyave

Núria Esteve-Gibert

Olaf Hauk

Oliver Quarrell

Onintza Sagredo

Owen Carmichael

Ozgul Gok

Paisit Paueksakon

Panagiotis Bamidis

Paola Rusmini

Paolo Calabresi

Patricia Broderick

Patricia K. Coyle

Patrícia Maciel

Patrizia Giannoni

Paul E. Greene

Paul Gorczynski

Paul Williamson

Peik Gustafsson

Peter C. Van Der Ende

Peter E. Wais

Peter N. Steinmetz

Peter Rumney

Philip Hazell 
Philippe Schucht

Phillip Tseng

Phu Hoang

Pierre Duquette

Pierre Roubertoux

Pieter J. Hoekstra

Pranela Rameshwar

Qingzhong Ren

Radwa Badawy

Rakesh Kumar Tiwari

Ramon Y. Birnbaum

Rany Makaryus

Rasheda Arman Chowdhury

Ray Meddis

Rebecca Fortgang

Rebecca Park

Remy Bation

Renee Hollingsworth

Reto Stocker

Ricardo S. Osorio

Richard C. Deth

Richard E. Mains

Richard Frye

Richard J. Youle

Rifai Chai

Robert B. Michael

Robert G. Mair

Robert Gonzalez

Robert Kotloski

Robert Lalonde

Robert R. Hansebout

Robert T. Mallet

Rocco Salvatore Calabro

Rodger J. Elble

Rodrigo Noseda

Romain Vuillefroy De Silly

Rosella Abeti

Rosemary Sheehan

Rupsa Datta

Russell Schachar

Ryan A. Stevenson

Samuel Frank

Sandy Shultz

Sanjay Maggirwar

Sarah Bayless

Sarah Hellewell

Sarah Lageman

Sarah Rabbitt

Scott R. Schroeder

Sebastian Gluth

Shashi S. Seshia

Shervin Assari
Shraddha Rege

Shyam Seetharaman

Signy Sheldon

Siobhain M. O'mahony

Soledad Zarate

Sonja Scholz

Sonya L. Jakubec

Sophia Frangou

Stan Kutcher

Stéphanie Chambaron-Ginhac

Stephen Holland

Stephen Honeybul

Stephen R. Jackson

Stephen Sawcer

Steven A. J. Chamuleau

Steven Passmore

Susan J. Nicolson

Susan Jerger

Susann M. Brady-Kalnay

Su-Youne Chang

Sven Kroener

T. Dianne Langford

Taku Hatano

Tatjana Aue

Terri Pogoda

Thanh Dang-Vu

Thérèse M. Jay

Thomas Heinbockel

Thomas W. Weickert

Tilman Hensch

Timothy J. Crow

Timothy Rittman

Tobias Derfuss

Tobias S. Andersen

Töres Theorell

Trinidad Montero-Melendez

Trishna Patel

Ulrich Palm

Vicki L. Kristman

Victor Rivera

Victor V. Dyakin

Victoria M. Leavitt

Vincent R. Harley

Vincenzo Guidetti

Vincenzo Natale

Virginia V. W. McIntosh

Vishwanath Sankarasubramanian

Wenchao Zhou

Wendy O. Kalberg

Wilhelm Mistiaen

William D. Kearns

Wissam Deeb 
Wolfgang Mann

Woo-Yang Kim

Xiaobing Yuan

Xueni Pan

Yadvinder Ahi

Yang Zhang
Ye Xiong

Yuan-Pin Lin

Yuri Bozzi

Yvonne Hoeller

Zhuo Xing

(C) 2018 by the authors; licensee MDPI, Basel, Switzerland. This article is an open access article distributed under the terms and conditions of the Creative Commons Attribution (CC BY) license (http://creativecommons.org/licenses/by/4.0/) 